\title{
Visualization of Multimodal Brain Connectivity for Neurosurgical Planning using Handheld Device Augmented Reality
}

\author{
Daiana R. Pur ${ }^{1}$, Denis Kikinov², Sandrine de Ribaupierre ${ }^{1,3}$ Roy A. Eagleson $^{1,2}$ \\ ${ }^{1}$ School of Biomedical Engineering \\ ${ }^{2}$ Department of Electrical and Computer Engineering \\ ${ }^{3}$ Department of Clinical and Neurological Sciences, Schulich School of Medicine and Dentistry \\ Western University, 1151 Richmond St, London, Ontario \\ dpur@uwo.ca; dkikinov@uwo.ca; sderibau@uwo.ca; eagleson@uwo.ca
}

\begin{abstract}
In neurosurgical procedures, precise preoperative planning requires extensive knowledge of the patients' anatomy as well as critical structures for brain functionality. Recently, there has been an increase in the use of minimally invasive approaches, owing in part to advancements in multimodal medical imaging techniques such as structural (SC), and functional-based brain mapping (FC), which have been shown to be useful metrics for surgical trajectory planning. The main challenges associated with their use is the lack of intuitive visualization and interactive methods available to neurosurgeons and trainees. AR systems represent a pivotal advancement towards augmenting the training of trainees as well as providing a platform for senior surgeons to maintain their skills in a low-risk training environment. Advanced image processing was performed on multimodal neuroimaging data (T1- weighted image, diffusion weighted image, resting-state functional magnetic resonance imaging) to characterize the SC and FC of the brain. An AR application, NeuroAR, was designed to take these as inputs and allow the user to visualize and interact with the neuroanatomy in the context of its associated SC and FC. The performances of 10 users on 24 targets were evaluated using an extension of Fitts' methodology. The users were able to use an interactive tool to select and visualize brain regions and their associated fibers. The fibers could be visualized based on their FC scores. As expected, the data showed that task difficulty increased as the volume of the fibers decreased. Movement time also increased as task difficulty increased. We introduced a new mobile device AR application based on data derived from advanced image processing of neuroimaging data. Evaluation of the 3D pointing tasks showed consistency in user performance indicating its utility.
\end{abstract}

Keywords: Augmented reality, Neurosurgery, Surgical planning, Multimodal imaging, Image fusion, Visualization, Surgical simulation.

\section{Introduction}

Numerous neurosurgical procedures require extensive multimodal preoperative brain mapping for identification of a precise surgical trajectory that will spare eloquent cortex (i.e. cortex that if damaged will lead to severe neurological deficits) and other critical structures. The introduction of minimally invasive surgeries, which require a small "keyhole" entry point into the skull, further emphasizes the need for the surgeon to be able to preoperatively visualize and interact with relevant brain structures to safely plan the neurosurgical trajectory. Conventionally, surgeons are required to do cognitively demanding mental transformations to coordinate between preoperative two-dimensional (2D) patient-specific magnetic resonance images (MRI) and patient reference frames (i.e. three-dimensional, 3D, anatomy) [1]. Additionally, they need to adeptly manipulate instruments in the surgical field while looking at a $2 \mathrm{D}$ display of preoperative images[2]. This is particularly challenging in the case of junior trainees who may have limited previous surgical experience and less developed spatial and perceptual intuition. Ultimately, this leads to longer surgeries and increases the chance of error associated with reduced performance due to cognitive overload[1].

Augmented reality (AR) incorporates patient-specific virtual preoperative data onto the user's vision of the real world[2],[3]. Medical applications of AR are on the rise in various fields of medicine both surgical (e.g. trajectory planning, training) and non-surgical (educational, psychiatric or psychological treatments). These have been extensively reviewed previously. [4]-[7]. AR use in neurosurgery includes AR systems for surgical planning, surgical navigation, or surgical training. These feature various methodologies and implementations (e.g. head-mounted display, augmented monitors, augmented optics) [8],[2]. Related work to NeuroAR, includes AR presurgical planning simulators aimed at facilitating training and planning of surgeries prior to the actual procedure, such as ImmersiveTouch[9], [10], a wearable Hololens device[11], mobile device AR applications [12], [13] (for review see[8], [14]). However, the majority of existing 
neurosurgery AR systems are devoted to intraoperative use for tumor resection, open neurovascular surgery, ventriculostomy, or spinal surgery[2]. Additionally, in contrast to NeuroAR, none of the surveyed systems made use of advanced image processing techniques to derive and display FC and SC information. The benefit of using multimodal brain connectivity measures in brain mapping is well established [15]. Therefore, FC and SC image fusion display in AR is of particular interest because it not only adds visuospatial context but also provides information about the importance of different brain areas based on preoperative scans (i.e. eloquence scores)[16], [17].

In this paper, we introduce a mobile device AR system, NeuroAR, designed and evaluated with human factors in mind, which allows real-time, intuitive, 3D visualization and interaction with brain structures and multimodal brain connectivity to facilitate training for neurosurgical procedures. Specifically, the individualized identification of eloquent cortex is facilitated by our system which displays both patient specific brain structural connectivity (SC) and functional connectivity (FC), as well as anatomical landmarks derived from MRIs. To evaluate the performance of the AR application, we extended Fitts' methodology and applied to a 3D pointing task guided by a 3D environment, presented on a mobile device screen (i.e. 2D display).

\section{Materials and Methods}

\subsection{MRI data acquisition and processing}

A functional resting-state fMRI (rs-fMRI) $\left(\mathrm{TE}=27 \mathrm{~ms}, \mathrm{TR}=2100 \mathrm{~ms}\right.$, flip angle $\left.=80^{\circ}, \mathrm{FOV}=200 \mathrm{~mm}\right)$, structural T1weighted image (time echo $[\mathrm{TE}]=2.27 \mathrm{~ms}$, time repetition $[\mathrm{TR}]=1900 \mathrm{~ms}$, field of view $[\mathrm{FOV}]=256 \mathrm{~mm}$, voxel size $1.0 \mathrm{x}$ $1.0 \mathrm{x} 1.0 \mathrm{~mm})$ and diffusion weighted image, DWI $(\mathrm{TR}=8400 \mathrm{~ms}, \mathrm{TE}=88 \mathrm{~ms}, \mathrm{~b}$ value $=1000 \mathrm{~s} / \mathrm{mm} 2$, and voxel size $2.0 \times$ $2.0 \times 2.0 \mathrm{~mm}$ ) were acquired from a healthy participant (30 years old, female). All the scans were acquired using a Siemens Trio 3T magnet. The MRI data were processed and then used as inputs in the AR application.

The T1-weighted image was analyzed using Freesurfer (v5.3) automated processing pipeline (http://surfer.nmr.mgh.harvard.edu/), to obtain the topological representation of the grey matter (i.e. cortical brain regions) in the form of a mesh brain surface. The standard steps for processing were implemented: motion correction[18], brain extraction using a watershed/surface deformation procedure [19], affine transformation (12 degrees of freedom) to the Talairach image space, non-uniform intensity normalization[20]. The brain was segmented into white matter (WM), grey matter (GM), and cerebrospinal fluid (CSF). Next, surface deformation following voxel intensity gradients to optimally place the grey/white and gray/CSF borders at the location where the greatest shift in intensity defines the transition to the other tissue class are performed to create a surface model of the brain [21]-[23]. Freesurfer's Desikan-Killiany Atlas was used to characterize the brain regions used in this study[24].

Next, 3D slicer (v4.9) (http://slicer.org) module Model Maker was used to create individual 3D VTK models of each brain region using each region's boundaries via a marching cube algorithm. The DWI was processed with FSL topup and eddy current correction to reduce artefacts (https://fsl.fmrib.ox.ac.uk/). 3D slicer modules DWI to DTI Estimation and Tractography Label Map seeding were used to generate fiber tracts of the WM (i.e. tractography) as VTK files using the DWI image. The algorithm matching brain regions to their WM fibers is based on MultiXplore (http://www.nitrc.org/projects/multixplore/), a scriptable module which can be added to 3D Slicer, previously discussed by Bakhshmand et al., (2017)[17]. Briefly, to obtain SC, WM fibers are clustered based on their intersection with cortical brain regions. FC was derived from the rs-fMRI, which was processed using an in-house pipeline composed of NiftiReg (http://cmictig.cs.ucl.ac.uk/wiki/index.php/NiftyReg) and FSL. The average of fMRI signals in each brain region was extracted, and a FC matrix was obtained.

\subsection{Augmented Reality processing}

For the 3D rendering of augmented reality, Unity was used with Vuforia Engine (http://unity3d.com): Unity: v2018.1.0, Vuforia: 7.2.24, any Android device running Android 7.0 or higher; tested on Samsung Galaxy S8+, and Samsung Galaxy Tab S2. Given the VTK (https://www.vtk.org) file format, a Unity-compatible model had to be generated. This was done manually, by using Paraview (https://www.paraview.org) to change each VTK file into a .x3d file and then through Blender (https://www.blender.org) by importing the .x3d files and exporting them as .obj files. To ensure WM fiber visibility, in Blender, the bevel attribute of the shape's geometry was adjusted (between 0.01 to 0.1 ).

Each model was individually brought into Unity and grouped together. For each fiber tract a button was created and connected to a brain region based on determined SC. These buttons were then added the functions to show or hide the associated fiber and connected regions. 
The FC matrix was also imported into Unity where it was parsed and saved during runtime. Using the parsed matrix data, the buttons for each fiber bundle were then coloured based on the correlation/connectivity strength of the two regions in the matrix, with dark red being at 1 , green at 0 , dark blue at -1 , with in-between values being coloured along a gradient.

A new Unity material with a custom vertex normals shader was created for use on the fibers. For each vertex, their normal was calculated and then translated into a colour: an RGB value between 0 and 1 . This gives the fibers a distinct colour based on their three-dimensional direction.

\subsection{Participants}

10 subjects participated in the experiment. The participants were university graduate students or undergraduate students. They all had normal or corrected-to-normal vision. All participants were novices in using AR devices and had minimal to no neuroanatomical knowledge. Therefore, 1 training trial was performed before engaging in the test tasks. Informed consent was obtained from all individual participants included in the study. All procedures performed in the study involving human participants were in accordance with the ethical standards of the ethics committee of the Faculty of Psychology and Educational Sciences of the University of Geneva and the Swiss Ethics committee, and with the 1964 Helsinki declaration and its later amendments.

\subsection{Evaluation Method}

A study consisting of a pointing task was used to evaluate the performance achieved by the AR application. Studies indicate that Fitt's law[25], a mathematical model used to describe the relationship between target size, distance, and movement time is a validated method for evaluating graphical interfaces and pointing tasks. First, an index of difficulty (ID) was calculate for each fiber tested, to evaluate the complexity of the pointing task. Index of performance (IP) was used to calculate movement time based on ID to indicate human performance. Human performance is defined as a trade-off between accuracy and speed.

$$
I D=\log _{2}(2 A / W)
$$

Where $\mathrm{A}$ is the distance between the center of the start cube and the center of the fiber, and $\mathrm{W}$ is the volume of the fiber.

$$
I P=\frac{I D}{M T}
$$

Where ID is the index of difficulty and MT is the movement time for the task.

\subsection{Experiment}

Unity with Vuforia were used for augmented reality rendering (Unity: v2018.1.0, Vuforia: 7.2.24), testing was done on Samsung Galaxy S8+, see Figure 1 for setup. Basic instructions about navigating the AR environment were given to the users. The users had a trial run to get comfortable using AR tool. Once the users were comfortable with interaction between the tool and the fibers, the testing began. Briefly, the users had to push a button "Perf Test", use the tool to collide with an AR cube, use the tool to touch the fiber, and finally touch any point on the screen when they were confident with their interaction. Upon clicking the button, the menu was hidden from the user, a single WM fiber was isolated along with the brain regions it connects and the start cube was generated. Besides touching the WM fiber and the cube nothing else can be

done, limiting random mistakes from users. Once the user touches the cube, it disappears giving the user the visual cue that the test has begun, where they are expected to touch the fiber; at this point data collection begins. When the user was certain they touched the fiber, they had to tap the phone screen with their finger, ending the data collection and the test for that fiber. This screen tap can only be done after the start cube has been touched, meaning that the user could not accidentally end the test before it began. The user repeated the process for $24 \mathrm{WM}$ fibers which were all logged. Once all the data had been saved, the original menu reappears signalling to the user that they have completed the task. The test fibers were chosen through an even distribution. All the fibers were pooled into a list and apart from the first fiber, were chosen based on their position in the list. The first fiber was randomly generated within the first $4 \%(1 / 25$ th) of the list. The rest of the fibers were chosen by going to the position relative to the test number, e.g. the second test was the fiber $2 / 25$ ths down the list. This resulted in the last fiber always being the last one in the list. To ensure the fibers were always the same, they were organized by originating 
brain region, and ensuring that no fiber was duplicated (there was only one fiber per two brain regions). For the pilot, a test size of 25 was decided, with 24 fibers being used to test the index of performance per user. After the user touched the start cube, data collection began, and a start time was recorded. Each frame, the position (3D, x, y, z in units used by Unity) and rotation (quaternion, $\mathrm{w}, \mathrm{x}, \mathrm{y}, \mathrm{z}$ ) of the needle relative to the fiber was logged, along with the time stamp. This resulted in position and rotational data being recorded about 60 times per second. Once the user completed the task by tapping the screen, the positional and rotational data was saved as a CSV file with the

associated task's number. Upon completion a second CSV file was generated which had the name of the fiber in the task, the calculated distance of the fiber from the start cube, the volume of the fiber and the total task time (time recorded at the end, subtracted by the start time). The volume of the fiber was calculated using the triangles and vertices of the fiber's mesh, resulting in larger fibers having larger volumes.

The test lasted approximately 15 minutes and could be done with a student population.

Fig. 1: The user's view during the pointing task through the mobile screen of the augmented reality application. Show LH, show left hemisphere; Show RH, show right hemisphere; Hide All, hide all brain regions; Show All, show all brain regions; Menu takes the user to the functional connectivity buttons.
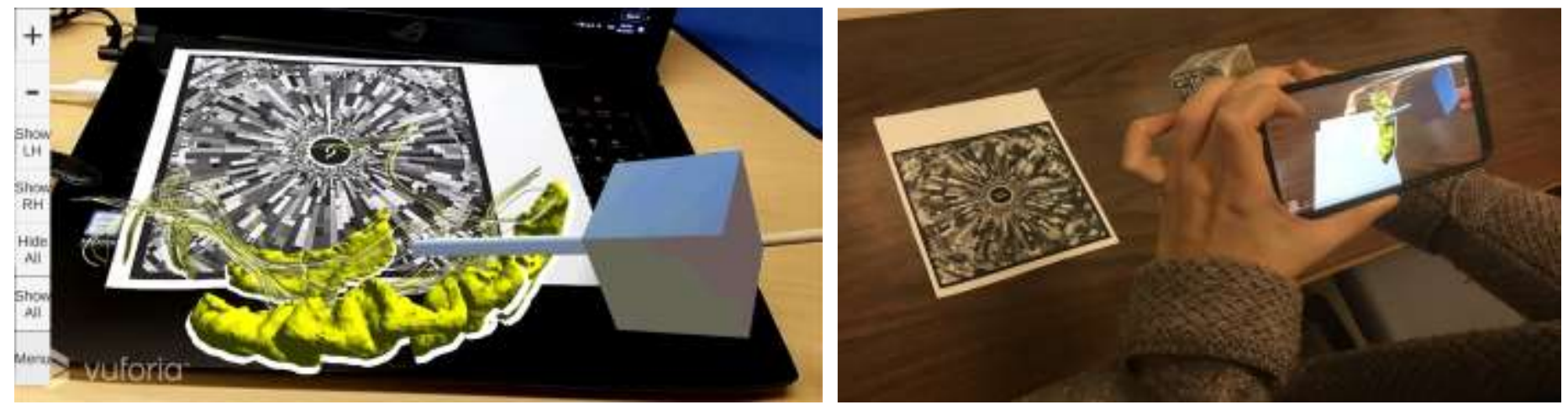

\subsection{Uses}

The user has many options on screen to choose from a) show, hide all the WM fiber tracts with their associated brain regions, b) individually show, hide WM fiber tracts, c) visualize the tractography (all WM fiber bundles) within the brain surface mesh or by itself, d) show, hide transparent brain surface mesh of the left and right hemispheres (see Figures 2,3). The background of the buttons on the menu are coloured based on FC. With these options the user is able to visualize as much of the tractography as they want, with or without the associated brain regions, and isolate certain clusters of brain regions of interest in the participant's brain.

There is also an option for the user to see which features are connected via interaction, where they can use an interactive tool to select different brain regions or the WM fibers between them.

Specifically, the user can use an interaction tool to touch the augmented model. If the tool comes into contact, via Unity's built-in collision system, with any WM fiber bundles it will highlight the contacted fibers as well as their source brain regions in yellow. This allows the user to physically test surgical paths and view which areas would be affected. At that point, the user is only able to interact with shown tractographies. 

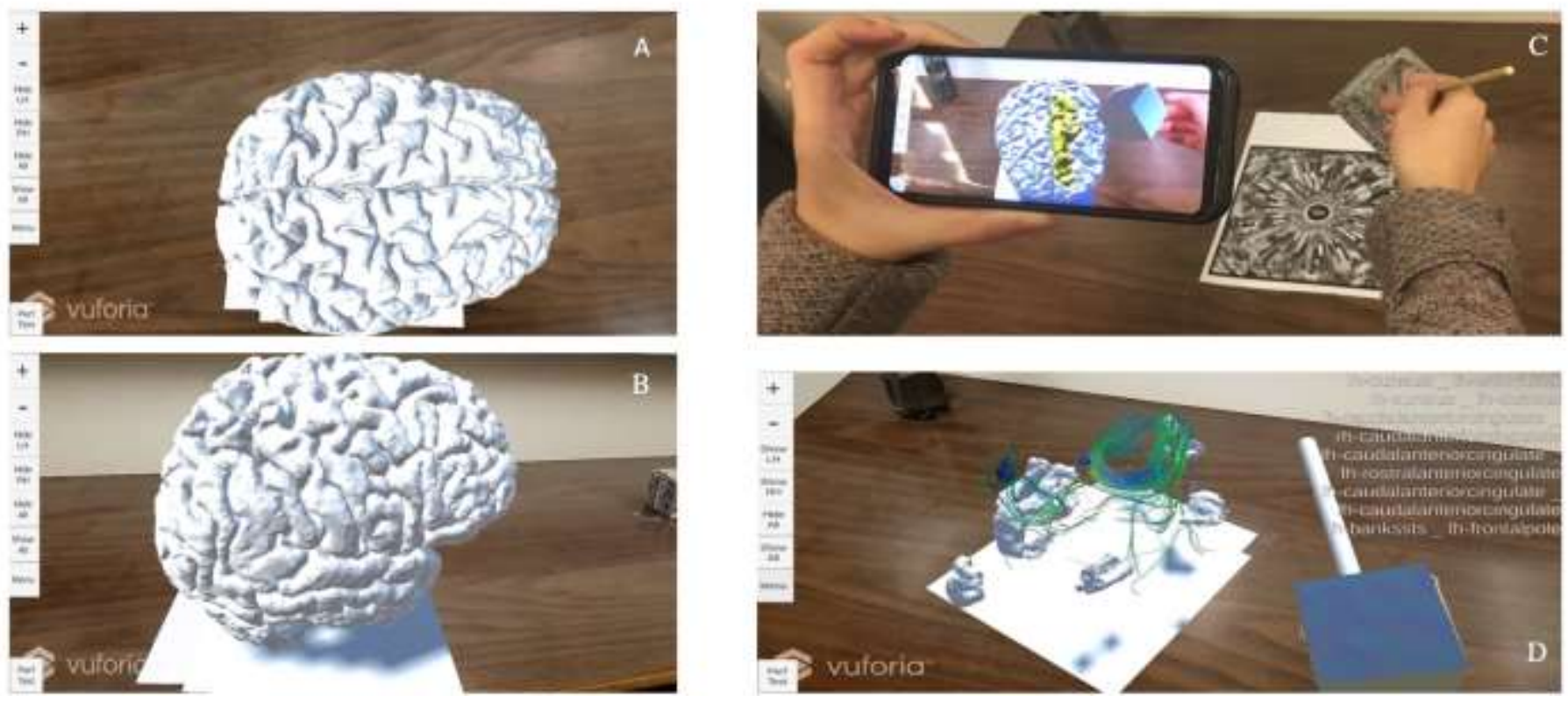

Fig. 2: The user's view through the mobile screen of the augmented reality application. The user can explore the neuroanatomy and structural connectivity of the brain using the tool. A) Axial view of the cortical surface mesh model of cortical grey matter. B) Sagittal view of the cortical surface mesh model of cortical grey matter. C) Depiction of user selecting a brain region on the cortical surface mesh model using the interactive tool. Region selected is yellow. D) View of the user exploring the structural connectivity between various regions (listed on the left side of the figure). Full model is hidden and only the brain regions selected are shown.

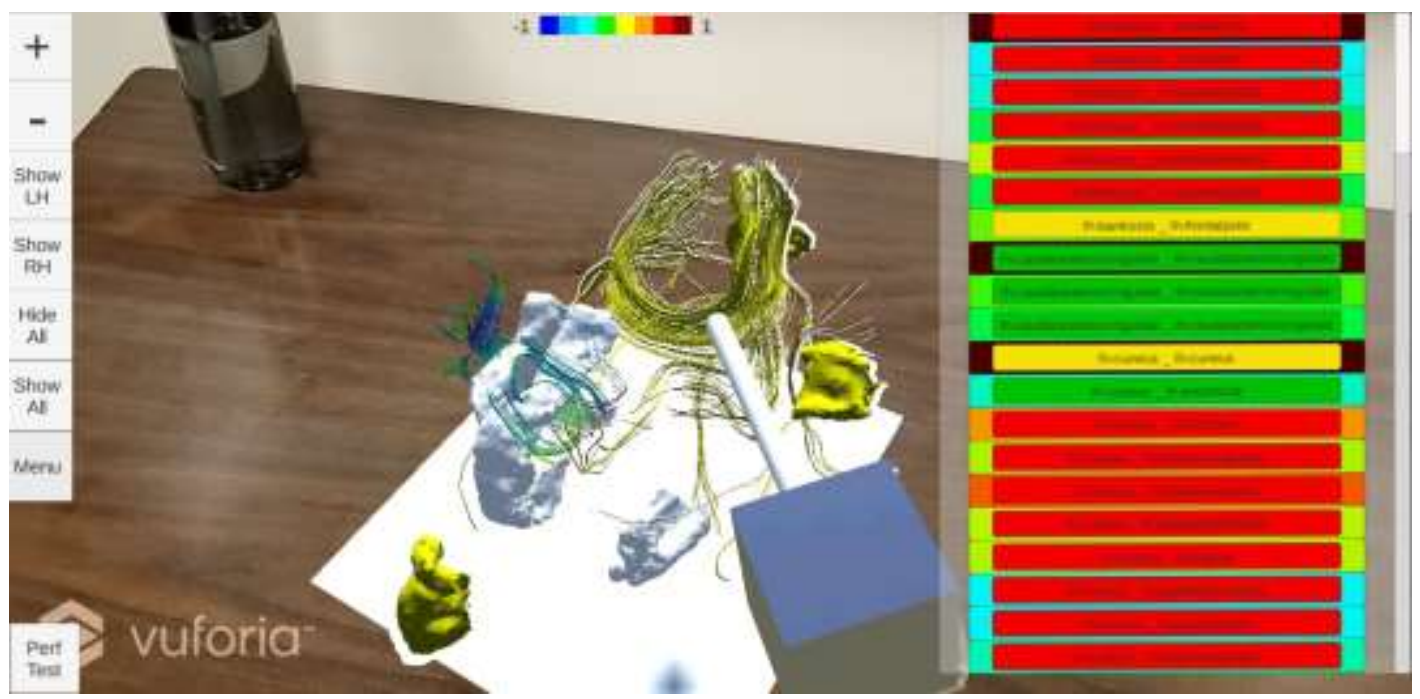

Fig. 3: The user's view through the mobile screen of the augmented reality application. The user can explore the neuroanatomy and both the structural and functional connectivity of the brain using the tool. Background of the buttons indicate the strength of the functional connectivity between the regions -1 strong negative, +1 strong positive. The user can explore these functionalities using the scroll bar.

\section{Results}

When using the AR application, anatomical detail (brain regions/structures) from the segmented T1 was able to be fused with a) the SC obtained from the processed DWI scan in the form of WM fiber bundles, b) the FC obtained from the processed rs-fMRI in the form of color-coded buttons. The buttons link two brain regions. 


\subsection{Performance test results}

Table 1, Figure 4 and Figure 5 indicate the outcome of the participants' trials in terms of the pointing tasks. Overall, our data showed that there was consistency in the responses of the users as depicted in Figure 5. As the volume of the WM fibers increased task difficulty decreased. As expected, as task difficulty increased so did movement time, depicted in Figure 4.

Table 1: Outcome of subjects' $(n=10)$ trials in terms of tasks $(n=24)$. For each pointing task/fiber target, the average and standard deviation of the performance of all subjects at that task was calculated. The volume and index of difficulty were constant across subjects and varied per task. MT, movement time; IP, index of performance; Volume, volume of the fibers; ID, index of difficulty.

\begin{tabular}{|c|c|c|c|c|c|c|}
\hline \multirow{3}{*}{$\begin{array}{c}\text { Task } \\
\text { no. } \\
1 \\
\end{array}$} & \multirow{2}{*}{\multicolumn{2}{|c|}{\begin{tabular}{lr} 
Avg & SD \\
\multicolumn{2}{c}{ MT (seconds) } \\
\end{tabular}}} & \multirow{2}{*}{\multicolumn{2}{|c|}{$\begin{array}{ll}\text { Avg } & \text { SD } \\
\left.\text { IP (u² }{ }^{2} \text { its/seconds }\right)\end{array}$}} & \multirow{3}{*}{$\begin{array}{c}\text { Volume }\left(\mathrm{u}^{3}\right) \\
0.009224 \\
\end{array}$} & \multirow{3}{*}{$\begin{array}{c}\text { ID }\left(\mathrm{u}^{2} \mathrm{bits}\right) \\
5.198\end{array}$} \\
\hline & & & & & & \\
\hline & 3.397 & \pm 1.302 & 1.698 & \pm 0.521 & & \\
\hline 2 & 7.787 & \pm 3.745 & 1.653 & \pm 1.257 & 0.000541 & 9.301 \\
\hline 3 & 5.233 & \pm 5.047 & 3.155 & \pm 1.524 & 0.000199 & 10.777 \\
\hline 4 & 3.318 & \pm 1.601 & 3.316 & \pm 1.247 & 0.000501 & 9.448 \\
\hline 5 & 9.451 & \pm 8.205 & 2.324 & \pm 2.120 & 0.000128 & 11.403 \\
\hline 6 & 11.321 & \pm 9.220 & 1.301 & \pm 0.876 & 0.000694 & 8.970 \\
\hline 7 & 5.258 & \pm 2.799 & 1.492 & \pm 0.679 & 0.004168 & 6.310 \\
\hline 8 & 5.340 & \pm 3.336 & 2.629 & \pm 1.862 & 0.000541 & 9.256 \\
\hline 9 & 3.013 & \pm 1.606 & 2.131 & \pm 0.971 & 0.010089 & 5.103 \\
\hline 10 & 6.522 & \pm 10.968 & 3.438 & \pm 2.720 & 0.00391 & 6.489 \\
\hline 11 & 4.940 & \pm 4.423 & 1.995 & \pm 1.484 & 0.009264 & 5.233 \\
\hline 12 & 7.970 & \pm 6.953 & 1.921 & \pm 1.606 & 0.00114 & 8.249 \\
\hline 13 & 5.455 & \pm 3.395 & 2.609 & \pm 1.341 & 0.000201 & 10.703 \\
\hline 14 & 11.360 & \pm 9.287 & 1.705 & \pm 1.151 & $5.77 \mathrm{E}-05$ & 12.494 \\
\hline 15 & 6.649 & \pm 9.244 & 3.920 & \pm 2.637 & $6.06 \mathrm{E}-05$ & 12.464 \\
\hline 16 & 4.333 & \pm 3.582 & 5.022 & \pm 2.855 & $2.45 \mathrm{E}-05$ & 13.827 \\
\hline 17 & 2.230 & \pm 1.386 & 5.145 & \pm 1.992 & 0.000547 & 9.306 \\
\hline 18 & 4.302 & \pm 2.223 & 2.666 & \pm 1.904 & 0.000968 & 8.519 \\
\hline 19 & 4.503 & \pm 4.692 & 2.730 & \pm 1.496 & 0.002858 & 6.965 \\
\hline 20 & 4.771 & \pm 3.030 & 4.500 & \pm 3.099 & $2.04 \mathrm{E}-05$ & 14.081 \\
\hline 21 & 4.108 & \pm 2.273 & 4.048 & \pm 1.787 & $3.45 \mathrm{E}-05$ & 13.298 \\
\hline 22 & 7.562 & \pm 5.814 & 1.984 & \pm 1.208 & 0.000425 & 9.620 \\
\hline 23 & 5.322 & \pm 3.536 & 3.008 & \pm 1.710 & 0.0001 & 11.723 \\
\hline 24 & 6.234 & \pm 4.895 & 3.070 & \pm 1.988 & 0.000117 & 11.457 \\
\hline
\end{tabular}



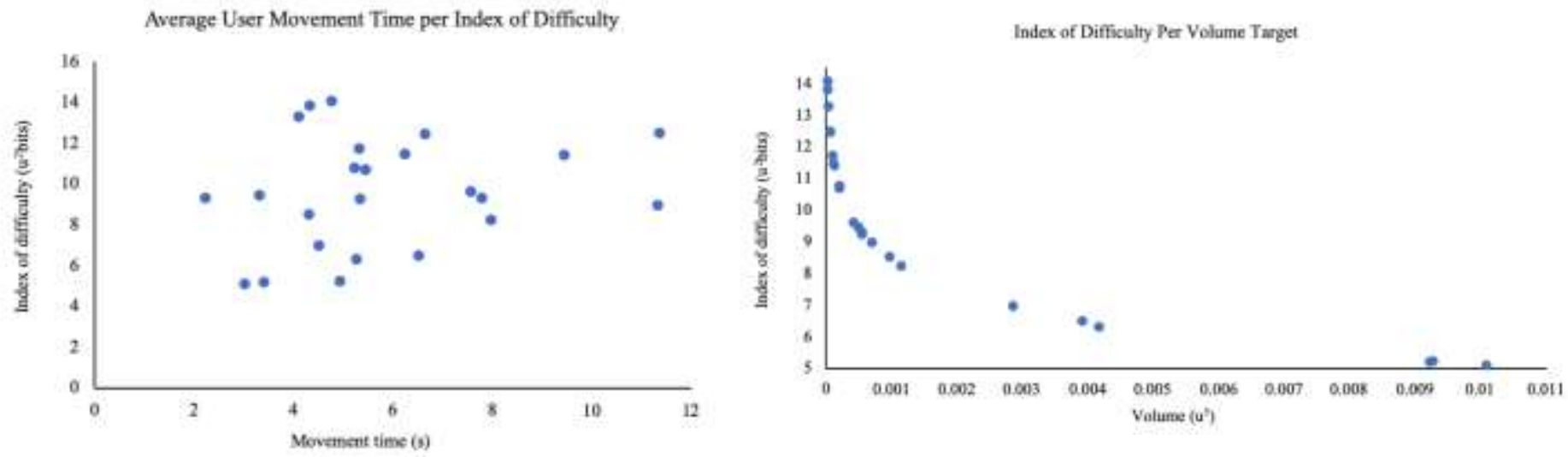

Fig. 4: Illustration of trends and relationships between various indices calculated according to Fitts Law. The values plotted represent the average response of the participants at each target.

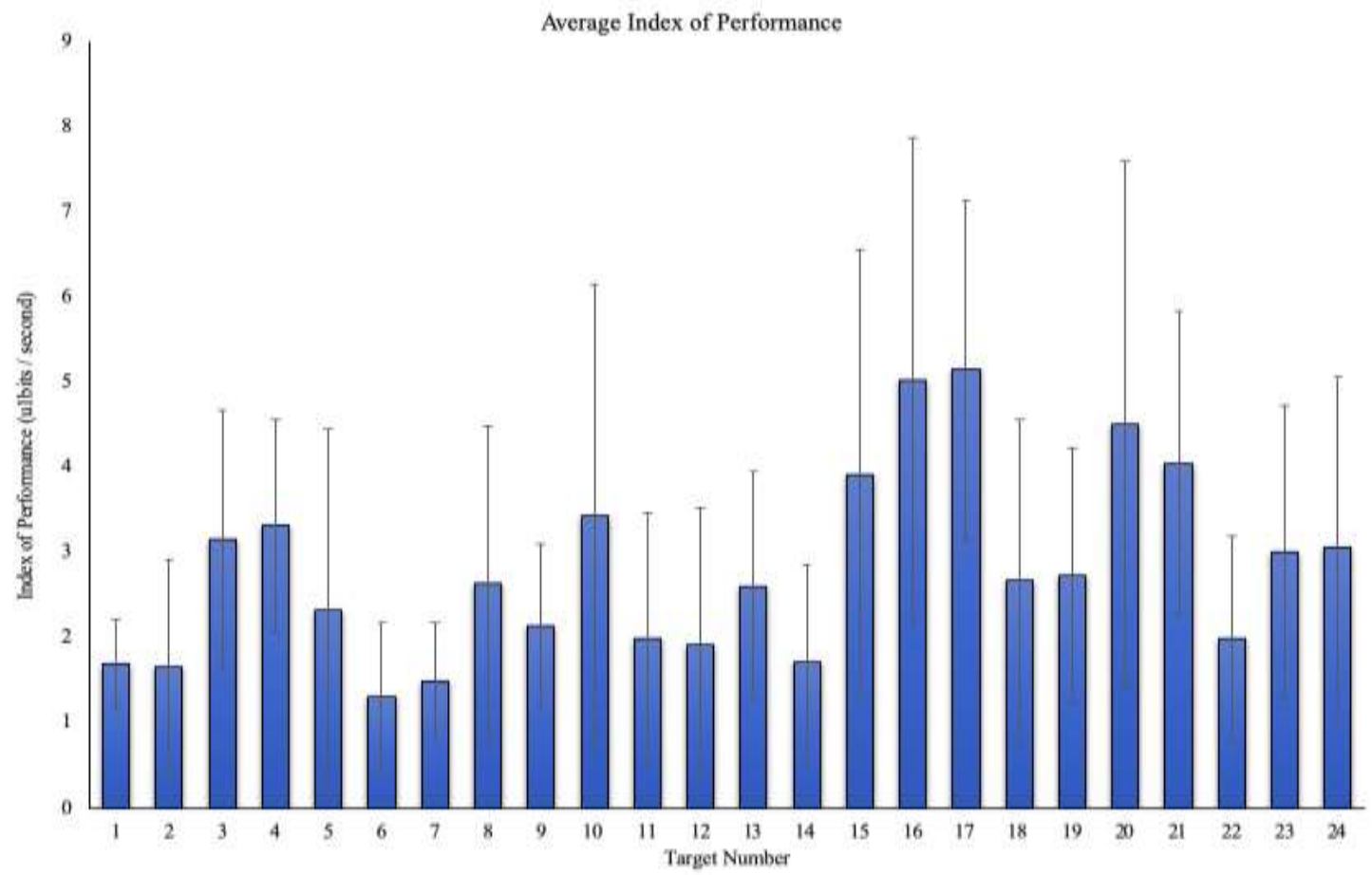

Fig. 5: Average index of performance of each user per pointing task/fiber target.

Based on the results of the performance test, the users found the application useable and were even able to interact with smaller fibers at the same speed as some of the larger ones. This is most notable with tasks 20 and 21 . The overall trends presented in Figure 5 show that most of the tasks were performed similarly among users, confirming the usability of the application. Any unexpected peak performances can be attributed to user error, as some users accidentally ended the task without touching the fiber, which led to higher standard deviation (most notable at task 10, 16, 20).

\section{Conclusion}

It is well established that minimally invasive approaches are beneficial to patient outcomes, and consequently it is crucial to maximize their safety and use. AR visualization and interaction with preoperative multimodal brain connectivity allows the surgeon to safely explore the patient anatomy and identify eloquent cortex based on advanced image processing, as well 
as try different neurosurgical approaches prior to surgery. Furthermore, our tool can also be used as a potential educational model for neurosurgical residents and medical students for neurosurgical planning. When tested amongst a pilot group of students, the users found the application useable and were able to interact with both small and large fibers without any issues. As expected, when targeting fibers with higher indices of difficulty, the movement time of the users increased, which is consistent to the extended Fitts' methodology.

\section{Acknowledgements}

This work was funded by NSERC operating grant R2680 to RE. The authors, Daiana R. Pur, Denis Kikinov, Sandrine de Ribaupierre, and Roy Eagleson declare that they have no conflict of interest.

\section{References}

[1] K. Abhari, J. S. H. Baxter, E. C. S. Chen, A. R. Khan, T. M. Peters, S. De Ribaupierre, and R. Eagleson, "Training for planning tumour resection: Augmented reality and human factors," IEEE Trans. Biomed. Eng., vol. 62, no. 6, pp. 1466-1477, 2015.

[2] D. Guha, N. M. Alotaibi, N. Nguyen, S. Gupta, C. McFaul, and V. X. D. Yang, "Augmented Reality in Neurosurgery: A Review of Current Concepts and Emerging Applications.," Can. J. Neurol. Sci., vol. 44, no. 3, pp. 235-245, May 2017.

[3] S.-L. Tang, C.-K. Kwoh, M.-Y. Teo, N. W. Sing, and K.-V. Ling, “Augmented realtiy systems for medical applications," IEEE Eng. Med. Biol., 1998.

[4] D. R. Berryman, "Augmented Reality: A Review," Medical Reference Services Quarterly. 2012.

[5] M. Harders, G. Bianchi, and B. Knoerlein, "Multimodal Augmented Reality in Medicine," in Universal Access in Human-Computer Interaction. Ambient Interaction, 2007.

[6] T. M. Rankin, M. J. Slepian, and D. G. Armstrong, "Augmented Reality in Surgery," in Technological Advances in Surgery, Trauma and Critical Care, 2015.

[7] I. A. Chicchi Giglioli, F. Pallavicini, E. Pedroli, S. Serino, and G. Riva, "Augmented Reality: A Brand New Challenge for the Assessment and Treatment of Psychological Disorders," Computational and Mathematical Methods in Medicine, 2015.

[8] A. Meola, F. Cutolo, M. Carbone, F. Cagnazzo, M. Ferrari, and V. Ferrari, "Augmented reality in neurosurgery: a systematic review," Neurosurgical Review, 2017.

[9] A. Alaraj, F. T. Charbel, D. Birk, M. Tobin, C. Luciano, P. P. Banerjee, S. Rizzi, J. Sorenson, K. Foley, K. Slavin, and B. Roitberg, "Role of cranial and spinal virtual and augmented reality simulation using immersive touch modules in neurosurgical training," Neurosurgery, 2013.

[10] C. Luciano, P. Banerjee, L. Florea, and G. Dawe, "Design of the ImmersiveTouchTM: a High-Performance Haptic Augmented Virtual Reality System," in Proceedings from International Conference on Human-Computer (HCI), 2005.

[11] F. Incekara, M. Smits, C. Dirven, and A. Vincent, "Clinical Feasibility of a Wearable Mixed-Reality Device in Neurosurgery," World Neurosurg., 2018.

[12] M. Kramers, R. Armstrong, S. M. Bakhshmand, A. Fenster, S. de Ribaupierre, and R. Eagleson, "Evaluation of a mobile augmented reality application for image guidance of neurosurgical interventions.," Stud. Health Technol. Inform., 2014.

[13] T. Wright, S. de Ribaupierre, and R. Eagleson, "Design and evaluation of an augmented reality simulator using leap motion," Healthc. Technol. Lett., 2017.

[14] P. E. Pelargos, D. T. Nagasawa, C. Lagman, S. Tenn, J. V. Demos, S. J. Lee, T. T. Bui, N. E. Barnette, N. S. Bhatt, N. Ung, A. Bari, N. A. Martin, and I. Yang, "Utilizing virtual and augmented reality for educational and clinical enhancements in neurosurgery," Journal of Clinical Neuroscience, 2017.

[15] H. B. pd. Shang, W. G. Zhao, and W. F. Zhang, "Preoperative assessment using multimodal functional magnetic resonance imaging techniques in patients with brain gliomas," Turk. Neurosurg., 2012.

[16] S. M. Bakhshmand, R. Eagleson, and S. de Ribaupierre, "Multimodal connectivity based eloquence score computation and visualisation for computer-aided neurosurgical path planning," Healthc. Technol. Lett., vol. 4, no. 5, pp. 152-156, 2017.

[17] S. M. Bakhshmand, A. R. Khan, S. de Ribaupierre, and R. Eagleson, "MultiXplore: Visual exploration platform for 
multimodal neuroimaging data," J. Neurosci. Methods, 2017.

[18] M. Reuter, H. D. Rosas, and B. Fischl, "Highly accurate inverse consistent registration: A robust approach," Neuroimage, 2010.

[19] F. Ségonne, A. M. Dale, E. Busa, M. Glessner, D. Salat, H. K. Hahn, and B. Fischl, "A hybrid approach to the skull stripping problem in MRI," Neuroimage, 2004.

[20] J. G. Sled, A. P. Zijdenbos, and A. C. Evans, "A nonparametric method for automatic correction of intensity nonuniformity in MRI data," IEEE Trans. Med. Imaging, vol. 17, no. 1, pp. 87-97, Feb. 1998.

[21] B. Fischl, M. I. Sereno, and A. M. Dale, "Cortical surface-based analysis: II. Inflation, flattening, and a surface-based coordinate system," NeuroImage, 1999.

[22] A. M. Dale and M. I. Sereno, "Improved Localizadon of Cortical Activity by Combining EEG and MEG with MRI Cortical Surface Reconstruction: A Linear Approach.," J. Cogn. Neurosci., vol. 5, no. 2, pp. 162-176, 1993.

[23] B. Fischl and A. M. Dale, "Measuring the thickness of the human cerebral cortex from magnetic resonance images.," Proc. Natl. Acad. Sci. U. S. A., vol. 97, no. 20, pp. 11050-11055, Sep. 2000.

[24] R. S. Desikan, F. Ségonne, B. Fischl, B. T. Quinn, B. C. Dickerson, D. Blacker, R. L. Buckner, A. M. Dale, R. P. Maguire, B. T. Hyman, M. S. Albert, and R. J. Killiany, "An automated labeling system for subdividing the human cerebral cortex on MRI scans into gyral based regions of interest," Neuroimage, 2006.

[25] P. M. Fitts, "The information capacity of the human motor system in controlling the amplitude of movement," J. Exp. Psychol., 1954. 